\title{
Teaching Astrobiology Online
}

\author{
S. T. Maddison
}

Centre for Astrophysics \& Supercomputing, Swinburne University, PO Box 218, Hawthorn, VIC 3122, Australia

\begin{abstract}
As part of Swinburne Astronomy Online ${ }^{1}$ (SAO), we run an online short course entitled Searching for Extrasolar Planets and Extraterrestrial Life. The main aim of the short course is to act as a "feeder" into our graduate programs and allow students to trial online education while exploring one of the new hot topics of astronomy - astrobiology.

I will present a brief overview of how SAO works, followed by an outline of our short course which has been running for four semesters. In particular, I will focus on why astrobiology is a good choice of topics for an online short course, and look at the successes (and failures) of the course in attracting students to both online education and astronomy and astrobiology in particular.
\end{abstract}

\section{Introduction}

Swinburne Astronomy Online (SAO) is a fully online graduate degree program in astronomy. SAO offers a nested degree program, consisting of a Graduate Certificate of Science, a Graduate Diploma of Science and a Master of Science by coursework. SAO is not a training program for $\mathrm{PhD}$ astronomers - it concentrates on the fundamental concepts of and key issues in contemporary astronomy, rather than its mathematical basis. SAO was initially designed for amateur astronomers, science educators and communicators, people working in astronomy related fields, and anyone with a love of astronomy.

The course material is delivered via custom-made CD-ROMs, each of which contains over 1500 animated slides written by professional astronomers, and accompanying multimedia material to enhance student learning. There are 15 units to choose from, with 12 units required for the Masters degree. Communication with instructors and fellow classmates is via asynchronous newsgroups and email, allowing students to study at a time that suits them. Assessment is via a mix of computer managed tests, an essay, a project and newsgroup contributions.

SAO has just completed its seventh semester, commencing world-wide delivery in March 1999. With the generous support of the Vice Chancellor of Swinburne University, SAO distributed a ClearSkies! CD-ROM with 200,000 copies of the January 2000 edition of Sky \& Telescope magazine, which helped

${ }^{1}$ http://astronomy.swin.edu.au/sao/ 

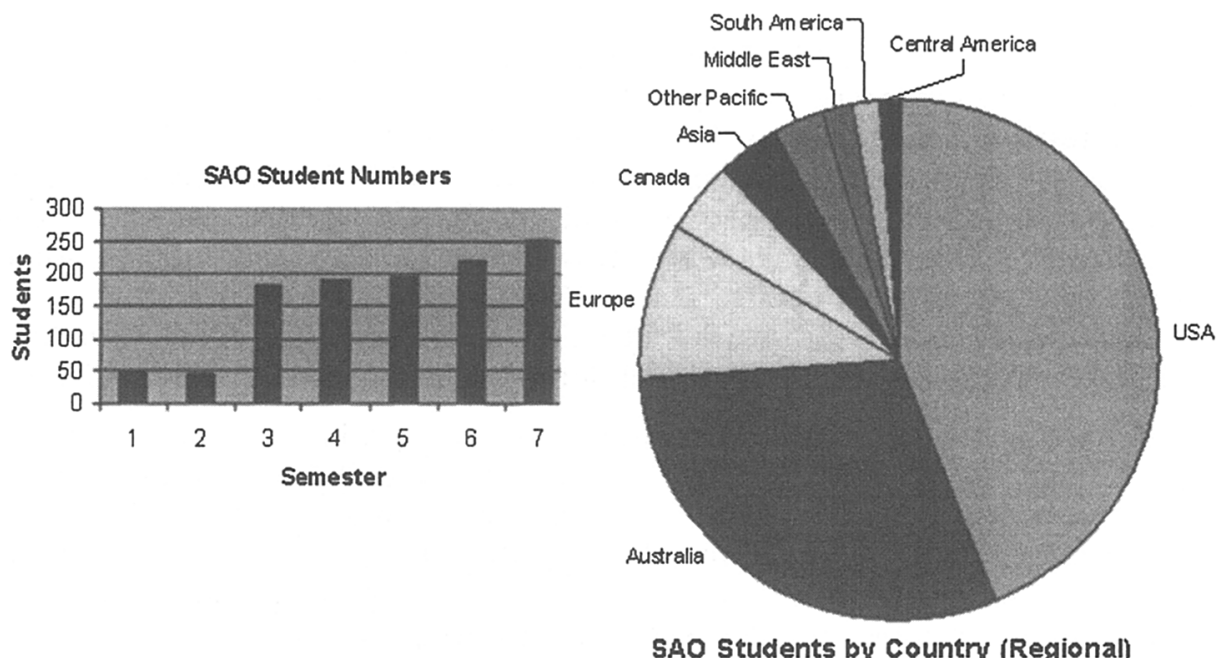

Figure 1. Student numbers against time (left hand side) and country of origin (right hand hide) of SAO students.

kick start the program. There are currently $20 \mathrm{SAO}$ graduates, including our first Masters students who graduated mid-2002.

SAO students numbers have steadily been increasing since its introduction, and it currently teaches into over 35 countries and has over 250 students taking more than 350 units per semester (see Fig. 1). About $30 \%$ of the students are from Australia, $40 \%$ from the USA, and $40 \%$ from over 30 other countries worldwide. Like most graduate astronomy courses, SAOs students are $20 \%$ female and $80 \%$ male.

\section{SAO Short Course}

The idea for an online short course in astronomy to compliment SAO was conceived in early 2000 . The purpose was two fold: (1) to feed students into the main SAO program who were apprehensive about online education and wanted to "test the waters" before enrolling in a full degree program; and (2) to accommodate students who did not want to or could not enroll in a graduate degree program but were interested in astronomy. Unlike SAO, the short course has no prerequisites - except a computer and internet access - and is open to people of any age and background knowledge.

After deciding to produce a 6 week online short course in astronomy, we needed to decide on a topic. We considered offering a general astronomy course, or a course specifically aimed at amateur observers, covering observational techniques and astrophotography. Such courses, however, are reasonably common and so we decided to go for a "sexy" hot topic in astronomy - the search for extrasolar planets and astrobiology.

The title of the short course is Searching for Extrasolar Planets and Extraterrestrial Life. The topics covered include: an introduction to star and planet 
formation; an overview of the Solar System; searching for extrasolar planets; understanding the new planets; an introduction to astrobiology; and the search for extraterrestrial intelligence.

For the short course, students download PowerPoint presentations each week and discuss the new topics in detail on the newsgroups. A list of relevant reading and internet links is also provided, as are some tempting questions for the students to think about.

But is it a good topic to teach? There are several excellent pedagogical benefits in teaching a course on the search for extrasolar planets and astrobiology:

- With new planets being discovered almost weekly, studying extrasolar planets clearly demonstrates that astronomy is indeed a very dynamic and exciting field. In addition, the physics involved in studying the orbital parameters of the extrasolar planets - Kepler's laws, gravity and conservation and angular momentum - is really quite neat and can show how a seemingly complex problem can be solved using quite simple mathematical equations.

- The search for extrasolar planets is a excellent example of a science experiment: extrasolar planets were theorised to exist, various techniques were devised to detect them, astronomers collected and analysed the data, and finally predictions were confirmed.

- Many areas of science can be taught, from basic physics such as gravity, to the truly interdisciplinary field of astrobiology which brings together many areas of science to solve a single (yet very complex!) problem - the origin of life.

- And students in general seem to love SETI! Here you can discuss sociology as well as technology.

The success (or failure) of the short course can be measured in three ways: by looking at the percentages of students who continue on to the main SAO degree program; by the student's feedback; and by monitoring the growth of the course.

The course has been running now for 4 semesters and to date our student numbers have been quite small - less than 60 students. This is most likely due to the lack of target advertising. (The fact that we offer a short course is mentioned in one bullet point of our SAO advertising, and is also on our SAO website.) However, the statistics we do have, along with the student feedback, tell us that: (1) just over $30 \%$ of short course students continue on to a degree program in $\mathrm{SAO}$; and (2) in general the students love the course. There are some that find online education is not suited to them and they are glad they did not enter the main program. Some feedback from our students include: "The breadth of information covered was amazing!"; "I was able to work at my own pace in my own time. The animations are very helpful in visualizing new concepts. I'd certainly recommend the course to others."

So while the SAO short course might not be considered a raving success in terms of student numbers, those that study the course seem to enjoy themselves, about a third continue on the main graduate programs, and it is indeed a pleasure to teach. Updating the material each semester can be a chore, but of course is all part of the excitement of astrobiology. 